\title{
Laying the groundwork for socialisation and knowledge construction within 3D virtual worlds
}

\author{
Shailey Minocha ${ }^{\mathrm{a} *}$ and Dave Roberts ${ }^{\mathrm{b}}$ \\ ${ }^{a}$ Centre for Research in Computing, The Open University, Milton Keynes, UK; ${ }^{b}$ Emerging \\ Technology Services, Warwick, UK
}

(Received 31 March 2008; final version received 8 September 2008)

\begin{abstract}
The paper reports the theoretical underpinnings for the pedagogical role and rationale for adopting 3D virtual worlds for socialisation and knowledge creation in distance education. Socialisation or 'knowing one another' in remote distributed environments can be achieved through synchronous technologies such as instant messaging, audio and video-conferencing. However, a 3D virtual world can provide an immersive experience where there is a visual presence and virtual proximity of the group members in terms of their 3D selves (avatars). We discuss the affordances of a 3D virtual world and its role in providing a platform for pedagogical design that engenders socialisation, synchronous communication and collaboration. We propose the use of a knowledge construction model as a framework for guiding the design of collaborative activities in a $3 \mathrm{D}$ virtual world for blended learning environments. We believe that this framework will also be useful for integrating 2D environments such as blogs, wikis and forums with a $3 \mathrm{D}$ learning environment. We consider the implications of this in the context of blended learning in distance education. This paper would be of interest to course designers, researchers, teachers, staff developers and policy-makers who are involved in integrating 3D virtual worlds within the curriculum of their programmes and institutions.
\end{abstract}

Keywords: 3D virtual worlds; online learning; socialisation; collaboration; synthetic worlds

\section{Introduction}

There are many schools of thought on learning, including behaviourism, cognitive psychology and constructivism, and no one theory is used exclusively for the design of networked learning environments. Course designers tend to include principles from all three (Anderson and Elloumi 2004). As research progresses, new learning theories to support the development of online materials are evolving. Over the last two decades, social theories of learning have assumed prominence in debate amongst researchers (e.g. Lave and Wenger 1991; Mayes 2001). Although the views of various social theorists differ (Salomon and Perkins 1998), there is a general consensus that interaction, dialogue and collaboration are essential for productive learning. Technology can provide a medium for conversing and collaborating within the learning environment (Jonassen, Peck, and Wilson 1999). The growing interest in social dimensions of learning has led to institutions adopting Virtual Learning Environments (VLEs) which have collaboration and communication tools such as wikis, blogs, forums and chat.

\footnotetext{
*Corresponding author: Email: s.minocha@open.ac.uk
} 
To integrate the social dimension in the pedagogy of online learning environments, Felix (2005) has proposed the synthesis of the cognitive and social constructivist approaches. In the cognitive constructivist approach, the focus is on the individual in the group, believing that cognition occurs in the head of the individual and that learners make intellectual sense of the materials on their own. The social constructivist approach emphasises the socially and culturally situated context of cognition, in which knowledge is constructed in shared endeavours (Duffy and Cunningham 1996). The interactions in the online environment, for example, through collaborations or discussions over forums, or in wikis, or on mailing lists, enable the knowledge to be constructed individually but mediated socially. The experiences of social interaction can be facilitated through interactive activities such as small group discussions, simulation games, project-based work, and collaborative problem-solving activities (Mason and Romiszowski 1996).

\section{Socialisation}

One important aspect of successful online experiences, student socialisation, appears to have a fractured research history and has been somewhat overlooked (Irwin and Berge 2006). However, research in online learning environments has shown that socialisation is, in fact, a central element in the student's experiences in collaborative activities. Salmon (2000) has developed a five-stage model for e-moderators. In the first stage the moderator addresses any technical or social issues that inhibit participation. In the second stage the moderator develops online socialisation by "building bridges between cultural, social and learning environments" $(2000,26)$. These early stages open the way for collaboration and knowledge construction (stages 3-5). Wilson (2001) has described that participants in online communities should have a shared sense of belonging, trust, expectation of learning, and commitment to participate and to contribute to the community.

For educators and students it is often difficult to assess one another's pre-dispositions in an online learning context and especially in the critical, early stages of the collaboration. Hence some course designers specifically give an opportunity at the start of students' learning interactions to share their understandings and particular aspects of themselves (Wegerif 1998). This sharing can be done formally, through questionnaires, but is often accomplished more effectively by ice-breaking activities. However, as we discuss below, studies have shown that:

- early socialisation in online learning environments may not be adequate to sustain the collaboration and co-operation (e.g. Minocha and Roberts 2008);

- the methods of socialisation such as general introductions and ice-breakers may not be sufficient for development of shared understanding amongst the learners (e.g. Irwin and Berge 2006); and

- socialisation may need to be integrated throughout the collaboration for development and sustenance of a learning community (e.g. Nicol, Minty, and Sinclair 2003).

In this paper, we first discuss the significance of socialisation in technology-enabled learning and the elements which constitute socialisation. We then discuss empirical evidence related to students' perceived lack of socialisation when collaborating in $2 \mathrm{D}$ environments such as blogs, wikis and forums. Next, the characteristics of a 3D virtual world are outlined and we discuss its role in providing a platform for pedagogical design that engenders socialisation, synchronous communication and collaboration. We propose the use of a knowledge construction model as a framework for guiding the design of collaborative activities in a 3D 
virtual world and for integrating $2 \mathrm{D}$ environments such as blogs, wikis and forums in conjunction with a $3 \mathrm{D}$ learning environment.

\section{Socialisation in technology-enabled learning}

Socialisation is of particular interest to psychologists, anthropologists, and sociologists. There are diverse opinions about its definition, the best methods for achieving it and effects it has on other outcomes. For the purposes of this paper, as applicable for technologyenabled distance education, socialisation encompasses the social act of coming together for a common purpose, for example, when students familiarise themselves with one other and learn about the norms, roles, rules and code of conduct. This initial phase of familiarisation is achieved through interacting with one another and sharing ideas and information. As a result of this shared experience, participants are able to become aware of one another, relate to one another (Wegerif 1998) and establish a connection. Socialisation, as a stage in developing collaborative learning in online environments, has been investigated in asynchronous 2 D environments such as in discussion forums (e.g. Salmon 2004; Motteram 2001).

Socialisation is a complex and dynamic aspect of education. Researchers in education, communication and media studies have discussed aspects that constitute socialisation. Irwin and Berge (2006) identified three elements of socialisation: interaction, presence, and knowledge construction. We will take each of these elements in turn to present the previous research in this area.

\section{Interaction}

Berge (1999) suggests that interaction is important to learner satisfaction and that it assists in maintaining student persistence in courses. There should be interaction between the learner and other learners, between the learner and the educator, and between the learner and experts. This allows them to collaborate, participate in shared cognition, form social networks, and establish social presence (Anderson and Elloumi 2004). The degree of interaction must be balanced to maintain student motivation. Too much interaction may be perceived as unhelpful and an overload, while too little interaction may result in student isolation (Berge 1999). Given that groups of students do not just become collaborative because they are assigned together (Johnson and Johnson 1996) and as dialogue is at the centre of teaching and learning (Laurillard 2002; Moore 1993), the online educator's most important responsibility is to ensure "a high degree of interactivity and participation" (Kearsley 2000, 78). One interesting view is that online interaction should seek to mimic a place-based meeting, to allow students the comfort of retaining face-to-face (FTF) conventions (Northrup 2002). Interaction is critical to create a sense of presence and a sense of community for online learners, and to promote collaborative learning (Murphy and Cifuentes 2001). The value of interaction lies in its contribution to the participant's ability to establish a sense of being in the virtual environment, that is, social presence.

\section{Social presence}

Social presence is defined as the degree of awareness of another person in an interaction and the consequent appreciation of an interpersonal relationship (Short, Williams, and Christie 1976; Rice 1993). Short, Williams, and Christie (1976) regarded social presence as the most important perception that occurs in an environment and stated that it is fundamental to person-to-person communication. Factors that contribute to the degree of social presence in 
a face-to-face encounter are facial expressions, direction of gaze, posture, dress, non-verbal cues, and vocal cues. In the absence of traditional communication cues in the electronic classroom, social presence is a measure of the feeling of community that a learner experiences in an online environment (Tu and McIssac 2002). Social presence, according to Shin (2002), is when students are "feeling intimacy or togetherness in terms of sharing time and place". Such a feeling of being connected to one another and reducing social distances is important when students are separated by geography (Brown and Duguid 2002) and directly affects the quality of interactions (Shin 2002, 126). There are a number of factors that influence social presence.

Privacy affects the degree of social presence. A less private setting results in a decreased perception of social presence by students. Champness (1972) reported that users felt more public in a video conference and perceived less social presence. Perse et al. (1992) found a positive relationship between social presence and a student's perception of their computer expertise. Social presence influences interaction and vice versa.

\section{Relationship between interaction and social presence}

Interaction includes the activities in which participants in a Computer-Mediated Communication (CMC) engage and the communication styles they use. Immediacy is a component of interactivity and is the psychological distance between a communicator and a recipient of the communication. In asynchronous $\mathrm{CMC}$, responses are delayed and a feeling of low immediacy and less awareness of interactivity can diminish social presence. Nicol, Minty, and Sinclair (2003) reported that students' experiences with asynchronous discussions were not positive because of time lag, passive participation ('lurking'), and absence of defined procedures or norms for contributions.

$\mathrm{Tu}$ and McIssac (2002) investigated the relationship between social presence and interaction in online environments. Tu and McIssac found several factors that influenced social presence in CMC: early familiarisation with recipients; timely response; developing trust (which requires more time in CMC); task types such as decision-making, creativity and social tasks, and perception of privacy. They found that social presence positively influenced online interaction. However, frequency of participation did not represent high social presence.

\section{Knowledge construction}

Social presence and interaction foster socialisation. Socialisation is the pre-requisite for effective collaboration and socialisation is also the outcome of collaboration, and hence, is pervasive throughout the collaboration. Knowledge construction is achieved by the interaction that takes place within oneself through reflective thinking and by the interaction that occurs in communications and collaboration with other people (Vygotsky 1978). The active exchange of ideas, dialogue and negotiation of views by looking at and reflecting on the multiple perspectives of fellow students facilitate collaborative and co-operative learning in which students and educators become active participants in the learning process.

In the next section, we discuss empirical evidence on the students' perceived lack of socialisation when collaborating in 2D environments.

\section{Perceived lack of socialisation in 2D environments}

Recent empirical research on the pedagogical role and effectiveness in the context of wikis (Minocha and Thomas 2007) and blogs (Kerawalla et al. 2008; Minocha and Roberts 
2008) in distance education has shown that inadequate socialisation at the start of the collaborative activity was a key obstacle in conducting group projects or activities at a distance.

In the post-graduate computing course (Minocha and Thomas 2008) which had a wikibased group project, the students do not meet face-to-face. It was only through online socialisation activities that the students got to know one another. Whilst there was an ice-breaker involving introducing one another in the wiki at the start of the group project, it was perceived to be inadequate by the students and several students commented on the difficulty of working with a group of relative strangers. One student stated:

Where project teams already know and understand each other, electronic communication is fine. Where strangers do not, all non-verbal communication is lost, leading to misunderstanding and potential conflict.

In a year-long research project (2006-2007) which investigated the role of blogs in learning and teaching, Kerawalla et al. (in press) examined two online courses. These are referred to as course ' $A$ ' and course ' $B$ ' in this paper. On course ' $A$ ', the usage of blogs was non-prescriptive and the course guide stated: "You will be encouraged to use personal blog (web log) as a learning journal to record and reflect upon your course activities, professional practices and development needs as you progress through the course". On this course, one of the student groups (18 students out of the 108 students registered on the course) was encouraged by their tutor to post photographs or anything personal such as poems, details of a recent holiday, and so on. Most students posted photographs of themselves, or of their family, or of family pets on the first few blog posts.

When the researchers asked the students in interviews about the benefits of posting these pictures and other personal artefacts, the students felt that exchanging personal artefacts was a good way to introduce themselves to the group with whom they would be collaborating. Comments on these early blog posts resulted in conversations, and a small group of students built up a support network through blogging to reach out to each other for both academic and social support (Minocha and Roberts 2008). Their posts were written in an informal, friendly style, and often addressed the reader directly. These posts contained high-quality academic work that was peppered with details of the students' personal life, photographs of their children, pictures to convey their moods or emotions, and also humour.

The socialisation via their blogs early in the course and sharing of personal thoughts and images throughout the course helped this small group of students to effectively collaborate and learn from one another. However, the majority of students on this course (those who were not encouraged to blog about personal aspects early on in the course) had concerns about blogging and stopped blogging after a few days into the course. Various social, pedagogical and technological factors influenced their perceptions about blogging (Minocha and Roberts 2008). Some of the students were anxious and self-conscious about blogging. They felt that their own inexperience as learners and users of technology, and their lack of confidence and potential incompetence, would be revealed to others in the public blogging space. The blogs had been administered by the university, and hence students were unable to make the blogs private or limit access to selected fellow students. The socialisation and community aspects of blogs hadn't been detailed in the guidance given to the students. One student could not see the purpose of the blog and how it fitted within the course. 
I couldn't actually see what use it would have or how I would use it. I couldn't picture it as a major part of the course at all.

On the online course B, early in the course the students were given a (compulsory) activity of posting the summaries of case studies and commenting on one another's blog posts. This activity was linked to the first assignment of the course. There was no formal or informal socialisation activity prescribed by the course or suggested by individual tutors. It was found that most students didn't blog at all after the first compulsory activity and some of them blogged mainly for themselves. The students on course B said that they had no choice other than to blog for themselves as the case-study activity had failed to stimulate any course blogging community (Kerawalla et al. in press).

Thus, even when tools such as blogs and wikis can facilitate socialisation, there is a need for educators to provide guidance on the role of the tool in socialisation and communitybuilding. Further, educators should integrate socialisation in the design of learning activities that encourage independent study which online learners prefer, and yet engender social presence, interactions and community-building through collaborative activities.

This recent empirical research on blogs and wikis, referred to in the papers mentioned here, uncovered drawbacks of lack of socialisation: inadequate peer review; hesitation to review critically or comment on each other's contributions; difficulties in negotiating tasks; and difficulty in scheduling the sub-tasks in the collaborative activity. Similarly, Nicol, Minty, and Sinclair (2003) found that students withheld their input in online exchanges because "they were uneasy about the impoverished social nature of online discussions" (274). However, even when wikis, forums and blogs can support socialisation, their asynchronous nature can affect students' perceptions of social presence and effectiveness of collaboration.

\section{Need for synchronous communication in collaborative projects}

Ehrlich and Chang (2006) have stated that co-ordination of work in distributed teams is accomplished through spontaneous informal communication. They also state that an important precursor to informal communication is awareness of other team members: awareness of what they are doing, and when they would be available for some aspect of the collaborative work. For ongoing collaboration in the wiki-based collaborative activity on the computing course discussed earlier (also see Minocha and Thomas 2007), over half of the students mentioned the need to engage in some form of synchronous communication for discussion and debate ranging from face-to-face sessions to telephone conferencing. One student said:

It is slow as a communication medium ... A wiki is not a flexible discussion medium.

I do not believe that a Wiki can be used in isolation when collaborative working, rather it should be adopted alongside other more traditional methods .e.g. telephone conferencing and face-to-face meetings ... face to face meetings should take place at various points in the requirements process in order to ensure that the process is managed correctly and difficult issues reviewed.

The students said that although the wiki had its strengths in recording decisions and supporting collaborative authoring, the wiki needed to be supported with a medium for synchronous discussion to facilitate timely decision-making. Indeed, face-to-face communication or other modes of synchronous communication can promote relationship-building (e.g. Peters 
and Manz 2007) and can generally help expedite negotiations and decision-making in collaborative activities. Face-to-face meetings are generally not feasible in distanceeducation courses. In fact, face-to-face meetings may sometimes interfere with the development of online communication skills (Salmon 2000) and, as we have seen, in online environments students' perceptions of privacy have an impact on social presence and they may not prefer face-to-face interactions. Further, the students on the two blogging courses and the wiki-based course discussed here were from around the world (including the UK, the USA and China) and, thus, it would not have been feasible to bring them together in a face-to-face setting.

Against this backdrop, we now discuss the role of 3D virtual environments such as Second Life in providing a real-life setting or 'place' for socialisation and its sustenance, collaboration and synchronous communication.

\section{D immersive virtual worlds}

$3 \mathrm{D}$ virtual worlds are multimedia immersive, ${ }^{1}$ simulated environments, often managed over the Web, which users can 'inhabit'. They can interact via their own graphical, humanoid, self-representations known as 'avatars'. Therefore, a virtual world is a cyberspace which has simulated bodies in simulated places. Castronova $(2005,11)$ prefers to use the term 'synthetic world' which he defines as an expansive, world-like, large-group environment made by humans, for humans, and which is maintained, recorded and rendered by a computer.

$3 \mathrm{D}$ virtual worlds are being used in many applications: education/training, gaming, social networking, marketing, and commerce. Some examples are: There.com and Activeworlds.com for social networking; World of Warcraft is a Massively Multi-player Online Role-playing Game (MMORPG); and Second Life, primarily for education and business. 3D virtual worlds dedicated to specific business domains are also being developed. For example, Forterra Systems have developed a virtual world called OLIVE (On-Line Interactive Virtual Environment). OLIVE enables customers to create virtual worlds primarily for training and simulations in healthcare, the military, and the media (Kushner 2008).

Unlike MMORPGs which have a scripted plot or storyline for the role-playing and game(s), Second Life, the 3D virtual world which we considered in this paper, is not a 'game' per se. It has a very strong user community, and the content and narrative is constructed and owned by the residents. In the following sections, we will discuss the role of $3 \mathrm{D}$ virtual worlds in socialisation and knowledge construction in online education by giving examples of Second Life because it the most widely used virtual world in education.

\section{D virtual worlds as learning environments}

The challenge for educators and course designers creating online education is to construct a learning environment that is simultaneously learning-centred, content-centred, community-centred, and assessment-centred (Bransford, Brown, and Cocking 1999). Similar to the 2D Web (Anderson and Elloumi 2004), the communication and collaboration characteristics of 3D virtual worlds facilitate the development of a learning environment which can support 'how people learn' (Bransford, Brown, and Cocking 1999):

- a 3D virtual world supports individualised and group activities through asynchronous and synchronous interactions in many formats (text chat, instant messaging, e-mail, voice chat, gestures); 
- it is knowledge-centred as the students can have direct access to vast libraries of content and learning activities in-world (within the 3D environment); and

- there are opportunities for formative and summative assessment by self, peers, and educators.

However, 3D virtual worlds, unlike the 2D Web, can offer immersion, realism and interaction through multimedia communication along with realistic, animated and customised avatars.

\section{Human social environment of virtual worlds: real-time interaction and social presence}

Using, audio, text and gestures the users, via their avatars, can converse in real time. Castronova (2005) says: "The physical environment is entirely crafted [in the synthetic world] and can be anything we want it to be, but the human social environment that emerges from that physical environment is no different from any other human social environment". Therefore, a 3D virtual world provides a social setting similar to a face-to-face setting where the relationships are being mediated by bodies (avatars) which are one step removed from the Earthly body, and therefore occur in a place that is one step removed from the Earth (Castronova 2005, 7).

Because of this, a 3D virtual world offers a sense of social presence via the avatars which may facilitate socialisation, an antecedent for effective operation of a virtual team in an educational context. This sense of presence and sense of place in a 3D world can make learning, and indeed socialising, in a virtual world a more 'human' experience than many other 2D environments. Further, a 3D virtual world enables creation of learning environments to enhance experiential learning, allowing individuals to practice skills, understand course concepts via 3D simulations and experiment with them, collaborate through roleplaying activities and have real-world-like experiences (e.g. Kamel Boulos, Hetherington, and Wheeler 2007). The lack of a guiding narrative in some virtual worlds such as Second Life provides flexibility to the educators and course designers to define an experience which matches the needs of the course. They can then design the learning space for that pedagogy rather than the other way around, which they may be forced to in some off-the-shelf VLEs. An 'island' in Second Life can provide a dedicated environment for learning which helps to ensure a sense of belonging and purpose for the students.

\section{Avatar-based communication}

3D virtual worlds facilitate communications which are different from chat, instant messaging, blogs, wiki, and the like. None of these tools invoke the Earth and a body as metaphors for interaction. 3D worlds allow people to experience human social life in an environment in which people interact with a body (avatar) they desire or choose. This feature of synthetic worlds removes from the social calculus all the unfortunate effects or any inhibitions that derive from the physical body (Castronova 2005).

Avatar-based communication is better than competing technologies such as instant messaging, blogs, wikis, forums, and so on at facilitating the interactions that humans want to have. 3D worlds provide a form of word-communication (through text and voice) and also enable a kind of bodily communication, through the gestures and position of the avatar. Thus they may offer a higher-quality forum of interaction than the chat room or the telephone, neither of which gives scope of the body. And while video conferencing gives both body and voice, it does not do so in a way that allows people to mingle with one 
another in a world-like space. Video conferencing can be very expensive in bandwidth. Avatar-based voice conferencing can provide a close substitute that costs much less. Thus, relative to these other communication tools, virtual worlds seem very competitive on the grounds of either better service or lower cost or both.

$3 \mathrm{D}$ virtual worlds should enable educators and learners to be more creative and to develop new effective ways of teaching and learning, rather than to purely replicate real-life classrooms in 3D virtual worlds. It is important to identify and focus on what 3D virtual worlds are most useful for: those activities that can only be effectively carried out in virtual worlds and not just as effectively in any other electronic medium. We must also determine and disseminate the optimal combination for blended approaches that combine 2D and 3D learning environments.

\section{Framework for design of Second Life activities}

In this section, we propose the application of a knowledge construction model as a framework for guiding the design of collaborative activities in a 3D virtual world and for integrating $2 \mathrm{D}$ environments such as blogs, wikis and forums in conjunction with a $3 \mathrm{D}$ learning environment.

\section{Knowledge creation model: Socialisation, Externalisation, Combination and Internalisation (SECI)}

Nonaka and Takeuchi (1995) proposed a model for knowledge creation: the SECI model involves conversion between explicit and tacit knowledge. The notions of explicit and tacit knowledge were introduced by Polanyi (1958) and used by Nonaka (1991) to formulate a theory of learning in an organisation that focuses on the conversion of knowledge between tacit and explicit forms. Explicit knowledge is represented by some artefact such as data, a document, a video, or a podcast, which has been created for communicating to another person and can be processed, stored and transmitted. In contrast, tacit knowledge is what the person possessing that knowledge 'knows', it is derived from experience and hard to formalise. Tacit knowledge is deeply rooted in action, procedures, routines, commitment, ideals, values and emotions. Knowledge is created through interactions between tacit and explicit knowledge, rather than from tacit or explicit knowledge alone (Nonaka, Toyama, and Konno 2000). Additionally, knowledge is created thorough interactions amongst individuals or between individuals and their environment. Through these interactions, tacit and explicit knowledge expands in both quality and quantity.

The SECI model encompasses the four modes of knowledge conversion and creation (Figure 1).

- Socialisation includes shared formation and communication of tacit knowledge between people through shared experiences. Socialisation may occur in apprenticeship or in informal social meetings where tacit knowledge such as world views and mental models can be exchanged and mutual trust and shared views can be created and shared.

- Externalisation is the process of articulating tacit knowledge into explicit knowledge. When tacit knowledge is made explicit, knowledge is crystallised, thus allowing it to be shared with others, and it becomes the basis of new knowledge. This process may involve performing activities which use metaphors, analogues, models, and the like, and result in creation of concepts. 


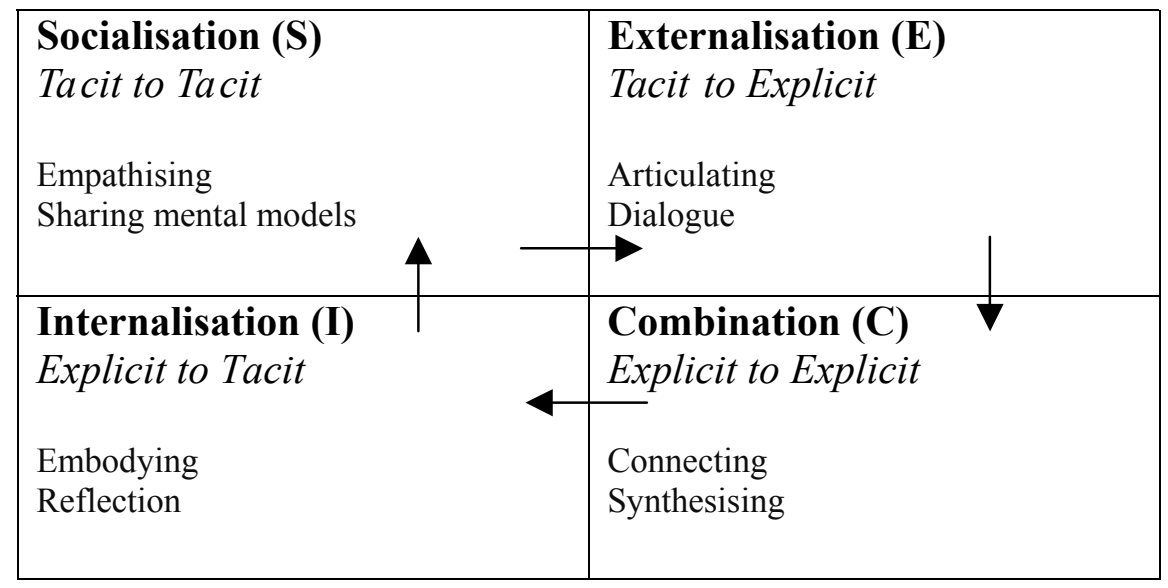

Figure 1. The knowledge creation model.

- Combination is a process of converting explicit knowledge into more complex sets of explicit knowledge. Explicit knowledge is collected from various sources within the group and from documents and websites outside the group to form new knowledge. This new knowledge is then disseminated among the members of the group in the form of a report.

- Internalisation is the process of embodying explicit knowledge into tacit knowledge. By reading documents and reflecting upon them, the explicit knowledge is internalised to enrich the tacit knowledge base. Explicit knowledge can also be embodied through simulations, or experiments that trigger learning by doing. This tacit knowledge accumulated at an individual level can then set off a new spiral of knowledge creation when it is shared with others through socialisation.

\section{Application of a SECI model: design of activities involving Second life and $2 D$ environments}

Using the SECI model, we will present the design of activities related to learning styles and skills development on a Level 1 distance education course in a university. This course is VLE-based and has a discussion forum, blog and wiki. The students meet their tutor in a face-to-face tutorial every other month. The motivation for introducing Second Life activities in this course has been inadequate participation of the students in the forums and poor attendance at the tutorials. The goal is to enhance the students' interactions and engagement with fellow students, their tutor and the university.

For the process of socialisation, the following activities will be performed by students:

- introduce themselves via their real names and not avatar names as these students work together in forums and other 2D environments on the course where real names are used;

- discuss the experiences on the course so far - one good and one bad aspect; and

- explore different parts of the university's Second Life island, guided via the teleport (location) map, and report back on one aspect of the island that really caught their attention. In the exploration of the island, the students could be specifically asked to 
hunt for objects in pairs on the island and report their location to the facilitator and the group.

In the second stage of the SECI model, the activity should involve discussion and articulation of knowledge. The students and the tutor (facilitator) will meet in Second Life for the activity. The facilitator will introduce the activity and various learning styles to the students. Based on the student's individual perceived learning style, the students will be asked to position themselves (their avatars) near a chart with displays of various styles. Students will then be encouraged to discuss at least one learning strategy that they use for their learning style. Next, they will be given a note card ${ }^{2}$ with characteristics of the learning styles based on theory (e.g., see http://www.chaminade.org/inspire/learnstl.htm). The students will be asked to discuss the learning styles in pairs and check whether there is any mismatch in their perceived learning style (what they thought) and the learning style that they actually have (based on characteristics derived from theory). The students will then come back together and position their avatars against the chart again. Then they will gather around as a group to report any mismatches to the group. This activity in Second Life will help to further socialisation and also to convert tacit knowledge into explicit knowledge.

After the activity in Second Life, students could discuss and record their perceptions and understanding of learning styles, and experiences of the collaborative activity in a 2D environment such as a wiki or a group-blog or a discussion forum (the process of combination of the SECI model).

Internalisation, the fourth process, involves reflection, for example by reading wiki or blog content, or writing about experiences as a part of the course work. Students could reflect on the collaborative activity and the collaboration process and record their reflections in their course work which will help internalise the knowledge of learning styles and the roles of learning styles in skills development. These reflections will facilitate learning and also provide useful feedback to the course designers about students' understanding of the task and their experiences and the effectiveness of the collaborative process.

The SECI model and the various tools involved in the sample activity are shown in Figure 2.

Some generic observations from the usage of the model in the design of this sample activity are: socialisation is integrated at every stage of the collaborative activity; and the SECI model can help guide the design of activities where usage of $3 \mathrm{D}$ virtual worlds can be combined with other $2 \mathrm{D}$ tools in the knowledge construction process.

\section{Discussion}

Socialisation is a key element of online learning and knowledge construction. Virtual icebreakers early on in collaboration may not be sufficient to develop and maintain shared understanding, mutual trust and social presence. Socialisation needs to be integrated and sustained in the collaboration process through the design of activities which are sure to reduce social distance amongst online learners. The various factors that influence socialisation are: interaction, synchronous communication, face-to-face-like social environment for communication and collaboration, and ensuring privacy of the learners.

\section{Blending of $2 D$ learning environments and media with $3 D$ virtual worlds}

3D virtual worlds like Second Life have the potential for providing a platform for learning, socialisation and knowledge creation - especially through providing social presence, 


\begin{tabular}{|c|c|}
\hline $\begin{array}{l}\text { Socialisation (S) } \\
\text { Tacit to Tacit } \\
\text { Ice-breakers in Second Life }\end{array}$ & $\begin{array}{l}\text { Externalisation (E) } \\
\text { Tacit to Explicit } \\
\text { Course activity in Second Life } \\
\underset{\text { Answering questions }}{\rightarrow}\end{array}$ \\
\hline $\begin{array}{l}\text { Internalisation (I) } \\
\text { Explicit to Tacit } \\
\text { Reflection: reading the blog } \\
\text { and wiki content; } \\
\text { Group-reflection in Second } \\
\text { Life sessions }\end{array}$ & $\begin{array}{l}\text { Combination (C) } \\
\text { Explicit to Explicit } \\
\text { Discussion and connection of } \\
\text { ideas in: blogs; group-blogs; } \\
\text { wikis; podcasts; vodcasts; } \\
\text { and Forums }\end{array}$ \\
\hline
\end{tabular}

Figure 2. Blended learning in a distance-education course involving 3D virtual worlds and 2D tools.

synchronous communication, privacy and interactions in a $3 \mathrm{D}$ environment via usergenerated avatars.

For effective knowledge construction, a 3D virtual world provides a space for collaboration. Extensive usage of a synchronous learning environment may be considered as an overload for students - especially those who are involved in part-time and/or distance education. Also, the students may require time for personal reflection. In conjunction with Second Life, the students will benefit from the provision of other tools such as a blog for reflection and note-taking, a wiki for collaborative authoring of an artefact, or a forum for asynchronous discussion.

The affordances of 2D Web and 3D virtual worlds are different but not mutually exclusive or a substitute for one another, but rather very complementary and synergistic. The challenges for educators are in designing activities that are situated within the pedagogical context of the course, meet students' needs of time and distance constraints, seamlessly integrate 2D Web and 3D worlds, deliver a positive student experience and, most importantly, meet the expected learning outcomes. Through some sample activities, we have shown the application of the SECI framework for the design of the activities involving 3D virtual worlds and 2D tools in the socialisation and knowledge construction processes.

Other synchronous technologies that are available in the educator's toolbox are audio or web conferencing tools such as Lyceum (http://lyceum.open.ac.uk) and Elluminate (http:// www.elluminate.com/) for real-time interaction and early socialisation in a course. However, the avatar-based real-time interactions in a 3D world provide a social setting almost equivalent to face-to-face. In addition, avatars can provide anonymity and privacy on aspects such as the physical appearance of the real person behind the avatar.

\section{Expressing emotions in avatar-based communication and collaboration}

In face-to-face conversations, our expressions signal our thoughts and feelings. A gaze indicates attention; narrowed lips reveal anger. The expressions are reliable means of 
communication, but we can also edit and control them. We feign attentiveness when bored and maintain a poker face during intense negotiations. Expressions that do not match our underlying feelings are essential not only for deception (Galanxhi and Nah 2007) but also for privacy and social graciousness.

Even though a 3D virtual world may provide a face-to-face environment, the expressiveness of current avatars is limited (Meadows 2008). The avatars can be moved next to each other to talk but often stare blankly into space, inert and unengaged. In current 3D worlds such as Second Life there is only a minimal set of user commands for expressing emotion, entered via the keyboard or selected from a menu. This minimal set of user interface controls and their lack of transparency (the controls can be difficult to interpret and interact with), has a potential to convey 'false' behaviour or 'no response' which may be unintended. Further, users may intentionally deceive by not conveying their actual emotional state (for example, giving a constant smile even when one is feeling very unhappy). Such false behaviours, intended or accidental, may affect aspects such as the interactions between the avatars in-world, the perceptions and reactions of the users on these interactions, and on the quality of the socialisation process.

Further, during a lecture in a face-to-face setting, it is common for educators to judge through visual cues about a student's presence in the class, their attentiveness, and whether they seem to understand what is being taught in the class. This enables the educator to change their teaching strategies to suit the situation. It is not possible for an educator to get these social and visual cues in an avatar-based communication and respond accordingly.

An important focus of research is, therefore, how to make avatars more expressive - able to make appropriate eye contact, smile, accurately show interest or boredom and so on (Donath 2008). Also, in the not-too-distant future, users will be able to choose the veracity of the avatars depending on the needs in each interaction, much as we now choose the communication media - video conference, phone, IM, e-mail - according to the nature of the message. Further, researchers predict that soon systems will allow the users the same revelatory and inhibitory control over the avatar's expressiveness that they have over their own (Donath 2008; Meadows 2008), thereby improving interactions in-world.

\section{Way forward}

Research is still needed to make 3D virtual worlds more accessible and usable. For effective design and conduct of activities in virtual worlds, learners, educators, and developers need to acquire and master new sets of competencies and skills in order for them to make the most efficient and effective use of 3D virtual worlds in learning and teaching.

Although in this paper we have demonstrated the application of the SECI framework, the next steps would be to introduce the framework to the community interested in adopting $3 \mathrm{D}$ virtual worlds in education along with 2D VLE tools. Systematic use and evaluation of the framework in our own institution and other institutions will help ascertain: (1) the benefits of the SECI framework in the design of activities on courses involving 2D and 3D environments; and (2) the role of 3D virtual worlds such as Second Life in socialisation.

In this paper, we have made an attempt to establish the link between socialisation, 3D virtual worlds and the pedagogical theories such as cognitive and social constructivism. However, the pedagogical underpinnings of socialisation and 3D virtual worlds need further investigation, specifically with regards to elements of socialisation such as presence and copresence and affordances of social and virtual proximity of 3D virtual worlds. One possible way would be to analyse the phenomenon of socialisation by applying the activity theory (for example, Engeström 2001 and Mercer 2000). 
Though the emotional aspects of 3D virtual worlds hasn't been the focus of our investigation, as the technology progresses to make avatars more expressive, there is a scope for further research. For example, to determine how easy it is for the sender to express his emotions and feelings via an avatar, how easy it is for the receiver to perceive these expressions and cues, and the impact of the avatar's expressiveness on the socialisation and collaborative learning.

\section{Notes}

1. The term 'immersion' is used to describe the psychological reaction of most users when they begin to use virtual worlds. At first they feel detached from the simulation, but after a very short period of time begin to experience the virtual world as though it were reality, i.e., they become 'immersed' in the experience (Roche 2007).

2. Note cards are the simple text documents that one can create and share in Second Life.

\section{References}

Anderson, T., and F. Elloumi. 2004. Theory and practice of online learning. Athabasca, Canada: Athabasca University. http://cde.athabascau.ca/online_book/pdf/TPOL_book.pdf.

Berge, Z.L. 1999. Interaction in post-secondary Web-based learning. Educational Technology 39, no. 1: 5-11.

Bransford, J., A. Brown, and R. Cocking. 1999. How people learn: Brain, mind experience and school. National Academy of Sciences Website: http://www.nap.edu/html/howpeople1.

Brown, J.S., and P. Duguid. 2002. The social life of information. Boston, MA: Harvard Business School Press.

Castronova, E. 2005. Synthetic worlds: The business and culture of online games. Chicago: The University of Chicago Press.

Champness, B.G. 1972. Attitudes towards person-person communications media. University College London, Unpublished Communications Studies Group Paper No. E/72011/CH.

Donath, J. 2008. Giving avatars emote control. The HBR List: Breakthrough ideas for 2008. Harvard Business Review 86, no. 2: 31.

Duffy, T.M., and D.J. Cunningham. 1996. Constructivism: Implications for the design and delivery of instruction. In Handbook of research for educational communications and technology, ed. D.H. Jonassen, 170-98. New York: Simon \& Schuster Macmillan.

Ehrlich, K., and K. Chang. 2006. Leveraging expertise in global software teams: Going outside boundaries. In Proceedings of the IEEE International Conference on Global Software Engineering (ICGSE'06), 149-58. IEEE.

Engeström, Y. 2001. Expansive learning at work: Toward an activity theoretical conceptualization. Journal of Education and Work 14, no. 1: 133-56.

Felix, U. 2005. E-learning pedagogy in the third millennium: The need for combining social and cognitive. ReCALL 17, no. 1:85-100.

Galanxhi, H., and F.F.-H. Nah. 2007. Deception in cyberspace: A comparison of text-only vs. avatar-supported medium. International Journal of Human-Computer Studies 65: 770-83.

Irwin, C., and Z.L. Berge. 2006. Socialization in the online classroom. e-Journal of Instructional Science and Technology 9, no. 1. http://www.usq.edu.au/electpub/e-jist/docs/vol9_no1/papers/ full_papers/irwin_berge.htm.

Johnson, D.W., and R.T. Johnson. 1996. Cooperation and the use of technology. In Handbook of research for educational communications and technology, ed. D.H. Jonassen, 1017-44. New York: Simon \& Schuster Macmillan.

Jonassen, D.H., K.L. Peck, and B.G. Wilson. 1999. Learning with technology: A constructivist perspective. Columbus, $\mathrm{OH}$ : Prentice Hall.

Kamel Boulos, M.N., L. Hetherington, and S. Wheeler. 2007. Second Life: An overview of the potential of 3-D virtual worlds in medical and health education. Health Information and Libraries Journal 24: 233-45.

Kearsley, G. 2000. Online education: Learning and teaching in cyberspace. Toronto: Wadsworth. 
Kerawalla, L.J., S. Minocha, G. Kirkup, and G. Conole. 2008. Characterising the different blogging behaviours of students on an online distance learning course. Learning Media and Technology 33, no. 1: 21-33.

- In press. Exploring student blogging activities in higher education. In Social software and developing community ontologies, ed. S. Hatzipanagos and S. Warburton. PA, USA: IGI Publishing.

Kushner, D. 2008. Make your very own virtual world with Olive. IEEE Spectrum 45, no. 1: $37-9$.

Laurillard, D. 2002. Rethinking university teaching: A conversational framework for the effective use of learning technologies. 2nd ed. London: RoutledgeFalmer.

Lave, J., and E. Wenger. 1991. Situated learning. Legitimate peripheral participation. Cambridge: Cambridge University Press.

Mason, R., and A.J. Romiszowski. 1996. Computer-mediated communication. In The handbook of research for educational communications and technology, ed. D. Jonassen, 438-56. New York: Simon \& Schuster Macmillan.

Mayes, T. 2001. Learning technology and learning relationships. In Teaching \& learning online: Pedagogies for new technologies, ed. J. Stephenson, 16-26. London: Kogan Page.

Meadows, M.S. 2008. I, Avatar: The culture and consequences of having a Second Life. California: New Riders.

Mercer, N. 2000. Words and minds: How we use language to think together. London: Routledge.

Minocha, S., and D. Roberts. 2008. Pedagogical effectiveness of wikis and blogs in E-learning environments. Pragmatics \& Cognition 16, no. 2: 272-306.

Minocha, S., and P. Thomas. 2007. Collaborative learning in a wiki environment: Experiences from a software engineering course. New Review of Hypermedia and Multimedia 13, 2: 187-209.

Moore, M.G. 1993. Theory of transactional distance. In Theoretical principles of distance education, ed. D. Keegan, 22-38. New York: Routledge.

Motteram, G. 2001. The role of synchronous communication in fully distance education. Australian Journal of Educational Technology 17, no. 2: 131-49.

Murphy, K.L., and L. Cifuentes. 2001. Using Web tools collaborating, and learning online. Distance Education 22, no. 2: 285-305.

Nicol, D.J., I. Minty, and C. Sinclair. 2003. The social dimensions of online learning. Innovations in Education and Teaching International 40, no. 3: 270-80.

Nonaka, I. 1991. The knowledge creating company. Harvard Business Review 69: 96-104.

Nonaka, I., and H. Takeuchi. 1995. The knowledge-creating company. New York: Oxford University Press.

Nonaka, I., R. Toyama, and N. Konno. 2000. SECI, Ba and leadership: A unified model of dynamic knowledge creation. Long Range Planning 33, no. 1: 5-34.

Northrup, P.T. 2002. Online learners' preferences for interaction. Quarterly Review of Distance Education 3, no. 2: 219-26.

Perse. E.I., P. Burton, E. Kovner, M.E. Lears, and R.J. Sen. 1992. Predicting computer-mediated communication in a college class. Communication Research Reports 9, no. 2: 161-70.

Peters, L.M., and C. Manz. 2007. Identifying antecedents of virtual team collaboration. Team Performance Management 13, no. 3/4: 117-29.

Polanyi, M. 1958. Personal knowledge. Chicago: University of Chicago Press.

Rice, R.E. 1993. Media appropriateness: Using social presence theory to compare traditional and new organizational media. Human Communication Research 19, no. 4: 451-84.

Roche, E.M. 2007. Crafting a strategy for virtual worlds: Eight questions to ask. The Conference Board - Executive action Series: http://www.barracloughltd.com/Welcome_files/Strategy_for_ Virtual_Worlds.pdf.

Salmon, G. 2000. E-Moderating: The key to teaching and learning online. London: Kogan Page.

. 2004. E-moderating: The key to teaching and learning online, 2nd ed. London: RoutledgeFalmer.

Salomon, G., and D. Perkins. 1998. Individual and social aspects of learning. In Review of Research in Education 23, ed. P.D. Pearson and A. Iran-Nejad, 1-24. Washington, DC: American Educational Research Association.

Shin, N. 2002. Beyond interaction: The relational construct of 'transactional presence'. Open Learning 17, no. 2: 121-36. 
Short, J.A., E. Williams, and B. Christie. 1976. The social psychology of telecommunications. London: Wiley.

Tu, C.H., and M. McIssac. 2002. The relationship of social presence and interaction in online classes. American Journal of Distance Education 16, no. 3: 131-50.

Vygotsky, L.S. 1978. Mind in society, the development of higher psychological processes. Cambridge, MA: Harvard University Press.

Wegerif, R. 1998. The social dimension of asynchronous learning networks. Journal of Asynchronous Learning Networks 2, no. 1: 34-49.

Wilson, B. 2001. Sense of community as a valued outcome for electronic courses, cohorts, and programs. http://carbon.cudenver.edu/ bwilson/SenseOfCommunity.html. 